\title{
Les cellules photovoltaïques organiques
}

Pierre Destruel (pierre.destruel@laplace.univ-tlse.fr) et Isabelle Seguy

Laboratoire Plasmas et Conversion d'Énergie, UMR CNRS 5213, Université de Toulouse III

La production

d'électricité d'origine

photovoltaïque pourrait

se baser sur l'utilisation

des matériaux organiques

\section{en complément}

du silicium.

La fabrication des cellules

photovoltaïques

organiques nécessite

une collaboration étroite

entre les chimistes

qui synthétisent

les matériaux,

les physiciens de l'état

solide qui les

caractérisent et

les ingénieurs qui

préparent les cellules

et qui mesurent leurs

\section{propriétés.}

Les termes suivis d'un astérisque sont définis dans le glossaire.

(1) Ici on ne parle pas de type $p$ et de type $n$, car cette dénomination est déterminée par la nature du dopant. Or, dans les organiques, les matériaux ne sont que très rarement dopés.

Plusieurs raisons justifient l'utilisation des matériaux organiques pour la fabrication des cellules photovoltaiqques. La plus importante se trouve dans les avantages particuliers de ces matériaux : ils peuvent être mis en forme facilement, par voie sèche (évaporation sous vide) ou par voie humide (Tournette, jet d'encre), avec des techniques simples, issues de l'industrie de la microélectronique. De plus, les quantités de matériaux utilisés sont relativement petites, les films ayant des épaisseurs de l'ordre de $100 \mathrm{~nm}$. Enfin l'ingénierie moléculaire permet d'adapter les valeurs du gap et des niveaux d'énergie $\mathrm{HOMO}^{\star}$ et LUMO^${ }^{\star}$. C'est pour cela que les recherches dans ce domaine sont très actives à travers le monde ; mais des problèmes restent à résoudre et ce type de cellule n'est pas encore commercialisé.

Une cellule PhotoVoltaïque Organique (OPV) est généralement fabriquée sur un substrat de verre recouvert d'ITO (couche mince - $150 \mathrm{~nm}$ - d'oxyde d'indium et d'étain possédant des bonnes propriétés de transparence dans le visible et de conductivité électrique). Ce substrat constitue l'anode du dispositif. Parfois, on remplace le verre par une feuille plastique souple recouverte également d'ITO. Les cellules ont des dimensions de quelques $\mathrm{cm}^{2}$, ce qui est suffisant pour les caractériser. Elles mettent en œuvre deux types de matériaux organiques qui ont des électronégativités différentes. Le plus avide d'électrons est qualifié d'accepteur (A), l'autre, celui qui cède facilement ses charges négatives, est appelé donneur (D). C'est une différence avec les cellules en silicium(1). Il est d'usage de classer les matériaux selon leur

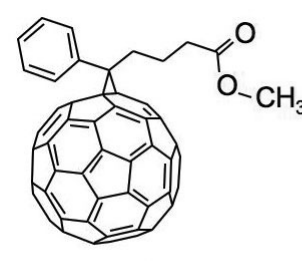

PCBM

Figure 1. Exemple de deux matériaux utilisés pour fabriquer une cellule photovoltaïque organique

Phenyl-C-Butyric acid Methyl ester (PCBM) comme accepteur et Poly(3-Hexyl)Thiophène (P3HT) comme donneur. masse molaire $M_{w}$. Pour des valeurs de $M_{w}$ élevées, on a des composés macromoléculaires (polymères), alors que pour des masses molaires plus petites, on les qualifie de matériaux moléculaires. Les polymères ne peuvent pas être déposés par évaporation sous vide. Un exemple de matériaux accepteur (PCBM, matériau moléculaire) et donneur ( $\mathrm{P} 3 \mathrm{HT}$, polymère) est donné sur la figure 1.

En 1978, les chercheurs de l'IBM Thomas J. Watson Research Center prévoyaient que les valeurs des rendements $\eta$ des OPV pourraient dépasser les $1 \%$ [1]. Ce rendement n'a été atteint que 8 ans plus tard, en 1986 [2]. Jusqu'en l'an 2000, cette valeur a parfois été reproduite [3], mais elle n'a jamais été améliorée. Ce n'est que depuis le début du XXI e siècle que le rendement a commencé à croître régulièrement, pour atteindre aujourd'hui les $6 \%$ [4]. Des modèles, basés sur les matériaux et les technologies actuellement utilisés, montrent qu'un rendement de $10 \%$ est envisageable à moyen terme [5].

Pour comprendre les stratégies de recherche mises en ouvre dans le domaine de la conversion photovoltaïque organique, il est nécessaire de connaître sommairement le fonctionnement d'une cellule. À cette condition, il est possible d'identifier les handicaps de ces composants et de proposer des solutions pour les contourner. Dans les OPV, le passage «du photon à l'électron " peut être décomposé en quatre étapes, représentées schématiquement sur la figure 2 dans le cas le plus simple où les films du donneur et de l'accepteur forment une jonction D/A planaire : 1) des excitons ${ }^{\star}$ sont créés au cœur des matériaux par absorption de photons ; 2) ils diffusent jusqu'à la jonction D/A où 3) ils sont dissociés en charges électriques libres; 4) ces charges rejoignent ensuite les électrodes.

\section{Absorption des photons et création d'excitons}

L'absorbance des matériaux organiques est égale au produit du coefficient d'absorption $\alpha$ par la distance d'interaction $l$ de la lumière dans le matériau (égale à l'épaisseur des couches pour des rayons lumineux perpendiculaires au plan des films). Elle doit être suffisamment grande 
pour que toute la lumière soit absorbée au travers de la cellule. Si $\alpha$ est généralement élevé ( $\left.\alpha>10^{5} \mathrm{~cm}^{-1}\right)$, l'épaisseur des films, de l'ordre de $100 \mathrm{~nm}$, conduit par contre à une absorption incomplète de la lumière incidente. Il est donc indispensable de chercher à augmenter la valeur de $\alpha$ par ingénierie moléculaire, mais également d'augmenter la valeur de $l$ en favorisant le couplage de la lumière dans les couches organiques. L'utilisation en face arrière d'une électrode qui possède une structure périodique [6], ou d'un réseau de Bragg [7], permet d'obtenir ce couplage recherché, comme le montre la figure 3 . La lumière est alors guidée dans le film organique et la distance d'interaction photons-matière devient grande. Enfin, des solutions originales comme la conversion de la lumière vers les longueurs d'onde fortement absorbées, ou encore la génération multiple d'excitons, sont étudiées pour atteindre des valeurs élevées du produit $\alpha l$.

L'absorption des photons dans les deux matériaux entraîne la formation d'excitons : des paires électron-trou corrélées par des forces de Coulomb. Au contraire du silicium (où l'énergie de liaison de l'exciton $\mathrm{E}_{\mathrm{L}}=14,7 \mathrm{meV}$ ), dans les organiques les excitons ne se dissocient pas à température ambiante, car la valeur de $\mathrm{E}_{\mathrm{L}}$ est beaucoup plus élevée, de l'ordre de quelques dixièmes d'électron-volt [8,9]. Les charges doivent être séparées par le champ électrique qui règne à la jonction $\mathrm{D} / \mathrm{A}$ entre le donneur et l'accepteur.

\section{Diffusion des excitons jusqu'à la jonction donneur-accepteur}

Les excitons ont une durée de vie $\tau$ très courte, de l'ordre de la nanoseconde. Si rien ne se produit pendant ce temps $\tau$, l'électron et le trou se recombinent et l'énergie de l'exciton se transforme en un nouveau photon ou en chaleur. Il faut donc dissocier les charges liées avant leur recombinaison. Les excitons ne sont pas localisés sur une molécule. Pendant leur durée de vie, ils diffusent sur une longueur $\mathrm{L}_{\mathrm{D}}$ dont la valeur est de l'ordre de quelques dizaines de nanomètres. Pour que les excitons puissent atteindre un site de dissociation (la jonction D/A), il faut qu'ils soient générés à une distance inférieure à $\mathrm{L}_{\mathrm{D}}$ de cette interface. Des études cherchent à augmenter la valeur de $\mathrm{L}_{\mathrm{D}}$ en induisant un ordre moléculaire dans les matériaux. Une alternative cherche à diminuer la distance que les excitons doivent parcourir pour rejoindre la jonction D/A. Pour cela, différentes configurations de cellules ont été proposées, comme les jonctions interdigitées ou interpénétrées. Elles sont schématisées sur la figure 4.

Enfin, il est possible d'augmenter la durée de vie des excitons en favorisant la conversion des singulets $(\mathrm{S})$ en triplets $(\mathrm{T})$ qui présentent une valeur de $\tau$ plus grande, pouvant atteindre quelques millisecondes [10]. Les excitons singulets sont formés par les électrons qui quittent le niveau $\mathrm{HOMO}\left(\mathrm{S}_{0}\right)$ et se placent sur un niveau énergétique de type $\mathrm{S}$. Par conversion intersystème, il peuvent se retrouver sur un niveau de type $T$, de durée de vie beaucoup plus grande. (La phosphorescence dure plus longtemps que la fluorescence.) Ainsi, les excitons disposent de beaucoup plus de temps pour se dissocier.

\section{Dissociation des excitons}

Lorsque les excitons rejoignent la jonction D/A, les deux charges qui les composent se séparent, à condition que l'une d'entre elles passe (soit transférée) de l'autre côté de la jonction. On obtient alors une paire de polarons ${ }^{\star}$ : un polaron de charge positive dans le donneur et un polaron de charge négative dans l'accepteur. Puisqu'il n'y a pas d'apport d'énergie au moment du transfert, celui-ci ne peut s'effectuer que si

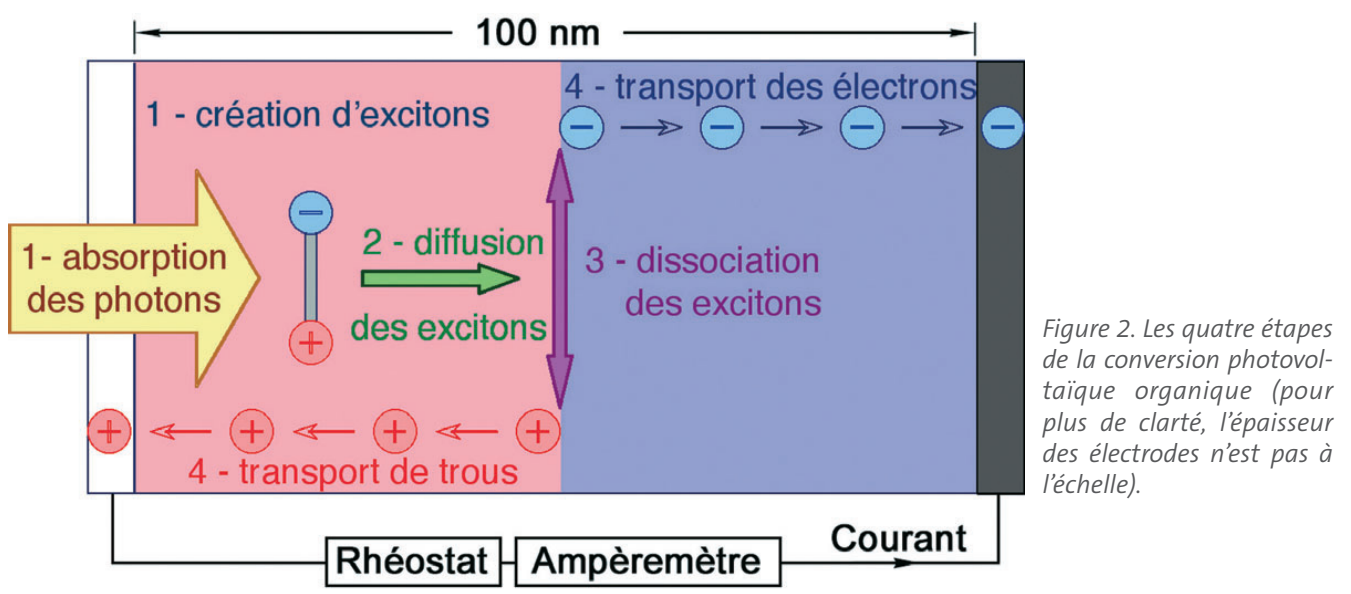

Figure 3. Couplage de la lumière dans une couche organique par la structuration périodique de la face arrière de l'électrode.
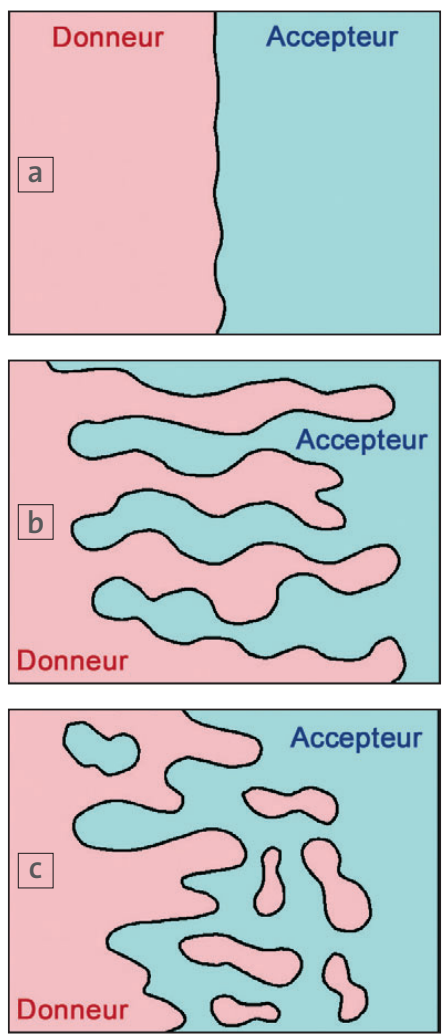

Figure 4. Trois types d'hétérojonctions rencontrées dans les cellules solaires organiques.

a) planaire $(\mathrm{PHJ})$; b) interdigitée $(\mathrm{IHJ})$; c) interpénétrée $(\mathrm{BHJ})$. Les interfaces interdigitées sont les plus difficiles à obtenir. Actuellement, la méthode proposée par l'Université de Princeton consiste à faire croître des aiguilles de phtalocyanine de cuivre (donneur) sur l'anode et à les noyer dans le matériau accepteur. D'autres méthodes sont à l'étude, par exemple celles basées sur l'auto-organisation des matériaux présentant une phase cristal liquide. Les interfaces interpénétrées sont obtenues par mélange du donneur et de l'accepteur. 


\section{Glossaire}

\section{HOMO}

Highest Occupied Molecular Orbital. L'orbitale moléculaire occupée la plus haute (en énergie) peut être comparée au haut de la bande de valence dans les semiconducteurs inorganiques.

\section{LUMO}

Lowest Unoccupied Molecular Orbital. L'orbitale moléculaire inoccupée la plus basse (en énergie) peut être comparée au bas de la bande de conduction dans les semiconducteurs inorganiques.

\section{Exciton :}

paire électron-trou liée par leur interaction coulombienne attractive. L'énergie de création de l'exciton, $E_{e x}$, est inférieure à l'énergie de bande interdite par une quantité $E_{L}$, dite énergie de liaison de l'exciton. On distingue deux types extrêmes d'exciton, celui de Frenkel, où la paire électron-trou est fortement liée, et celui de WannierMott où la distance entre électron et trou est grande par rapport à la distance interatomique et donc l'énergie de liaison est faible. Dans les matériaux organiques, les excitons sont plutôt de type Frenkel.

\section{Polaron}

ensemble d'une charge électrique associée au champ de déformation de son environnement proche, sous l'effet des forces électrostatiques qu'elle engendre.

\section{Références}

\section{[1] V.Y. Merritt, IBM J. Res.}

Develop., 22 (1978) 353.

[2] C.W.Tang, Appl. Phys. Lett., 48 (1986) 183

[3] D. Wöhrle et D. Meissner, Adv. Mater., 3 (1991) 129.

[4] Kyungkon Kim et al., Appl. Phys. Lett., 90 (2007) 163511.

[5] M.C. Scharber et al., Adv. Mat., 18 (2006) 789.

[6] C. Cocoyer et al., Appl. Phys. Lett., 88 (2006) 133108.

[7] L. Zeng et al., Appl. Phys.

Lett., 89 (2006) 111111.

[8] G. Hill et al., Chem. Phys.

Lett., 327, 3-4 (2000) 181

[9] M. Knupfer, Appl. Phys. A

Mat. Sci. \& Proc., 77 (2003) 623.

[10] C-M.Yang et al., Appl. Phys.

Lett., 90 (2007) 133509.

[11] P. Peumans et al., J. Appl.

Phys., 93, 7 (2003) 3693

[12] J.O. Haerter et al., Appl. Phys. Lett., 86 (2005) 164101.

[13] O.D. Jurchescu et al., Appl. Phys. Lett., 84, 16 (2004) 3061. [14] http://www.solaireorganique.ups-tlse.fr/

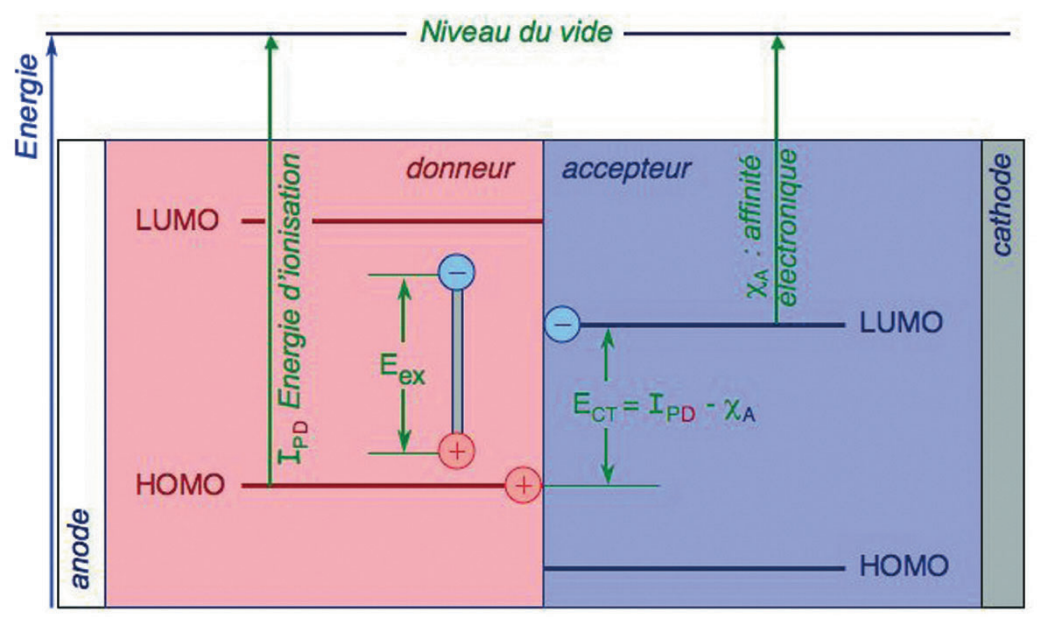

Figure 5. La condition de dis sociation des excitons à la jonction donneur/accepteur. La figure est dessinée pour un exciton créé dans le donneur. Une figure équivalente peut être tracée avec l'exciton dans l'accepteur et le passage du trou vers le donneur.

Pour obtenir la dissociation: $\mathrm{E}_{\mathrm{CT}}=\mathrm{I}_{\mathrm{PD}}-\chi_{\mathrm{A}}<\mathrm{E}_{\mathrm{ex}}$

l'énergie finale de la paire de polarons $\mathrm{E}_{\mathrm{CT}}$ est inférieure à l'énergie initiale de l'exciton $\mathrm{E}_{\mathrm{EX}}$ [11] En d'autres termes, l'énergie de l'ensemble des deux charges séparées doit être plus petite que celle de l'exciton. Cela entraîne une condition, indiquée sur la figure 5, sur les positions relatives des niveaux HOMO et LUMO des deux matériaux organiques. Ici encore, l'ingénierie moléculaire peut aider à satisfaire cette condition.

\section{Transport des charges et collecte aux électrodes}

Dans cette quatrième étape, les porteurs de charge doivent rejoindre les électrodes en traversant les films organiques. Le manque d'ordre atomique dans les matériaux organiques est une autre différence importante avec les cellules en silicium cristallin. Ainsi, les mobilités des électrons $\mu_{\mathrm{e}}$ et des trous $\mu_{\mathrm{h}}$ sont faibles. Un objectif de la recherche actuelle est d'augmenter leurs valeurs en induisant un certain ordre dans les matériaux. Il a été montré qu'elles doivent être au moins égales à $10^{-3} \mathrm{~cm}^{2} \mathrm{~V}^{-1} \mathrm{~s}^{-1}$ pour des épaisseurs de film de $100 \mathrm{~nm}$ [12]. De plus, il est important que leurs valeurs soient voisines pour les deux matériaux D et A utilisés, de façon que les charges n'arrivent pas plus vite à une des deux électrodes. Les valeurs des mobilités dépendent de nombreux facteurs. Parmi les plus importants on peut citer les pièges (d'origine chimique, liés à la structure, aux impuretés...), mais également la différence d'énergie entre les niveaux LUMO et HOMO (le gap)... Il a été montré que, dans le pentacène, une mobilité de $35 \mathrm{~cm}^{2} \mathrm{~V}^{-1} \mathrm{~s}^{-1}$ pouvait être calculée à partir des caractéristiques $\mathrm{I}(\mathrm{V})$ à température ambiante, après purification du matériau [13]. Lorsque les porteurs ont traversé les couches organiques, ils arrivent aux interfaces avec les électrodes. Ils doivent être collectés pour rejoindre le circuit électrique extérieur.
Des précautions doivent être prises pour éviter une diminution de la tension de circuit ouvert $V_{\text {OC }}$ et une résistance série $R_{S}$ trop élevée.

\section{Conclusion}

" La solidité d'une chaîne repose sur la solidité du plus faible maillon. " Il en est de même pour la conversion photovoltaïque organique. Si l'une des quatre étapes décrites ci-dessus est défaillante, l'ensemble du processus de conversion en est affecté et le rendement de conversion de la cellule reste médiocre.

Pour construire des cellules photovoltaiques organiques dont les performances permettront leur utilisation pour produire de l'énergie, les handicaps qui apparaissent à chaque étape doivent être contournés.

Une cellule ne sera économiquement viable que si le prix du kilowatt-heure produit est concurrentiel avec les autres sources d'énergie renouvelable. Pour minimiser ce paramètre, le module photovoltaïque et son entretien devront êtres les moins chers possible. Cela entraîne des conditions sur la durée de vie minimum des cellules. Or les matériaux organiques sont réputés pour leur vieillissement rapide en condition d'éclairement moyen au sol (AM 1,5, voir encadré p. 7 de Reflets n5). L'encapsulation, mais aussi l'ingénierie moléculaire et le contrôle de la stabilité de la morphologie des matériaux, devront être étudiés pour obtenir une stabilité satisfaisante des cellules.

Les handicaps sont sévères, mais la marge de progression est grande. Si aucune certitude n'est aujourd'hui raisonnable quant au succès futur de ces cellules, les enjeux sont suffisamment importants pour continuer les efforts de recherche dans ce domaine. En France, le réseau CNRS-CEA Nanorgasol [14] a pour objectif d'induire une synergie entre tous les laboratoires de recherche qui travaillent dans ce domaine. 